\title{
Article
}

\section{Odd Radio Circles and Their Environment}

\author{
Ray P. Norris ${ }^{1,2, *(\mathbb{D}, \text { Evan Crawford }}{ }^{3}(\mathbb{D})$ and Peter Macgregor ${ }^{1,2} \mathbb{D}$ \\ 1 School of Science, Western Sydney University, Locked Bag 1797, Penrith, NSW 2751, Australia; \\ P.Macgregor@westernsydney.edu.au \\ 2 CSIRO Space \& Astronomy, P.O. Box 76, Epping, NSW 1710, Australia \\ 3 School of Computer, Data and Mathematical Sciences, Western Sydney University, Locked Bag 1797, \\ Penrith, NSW 2751, Australia; E.Crawford@westernsydney.edu.au \\ * Correspondence: raypnorris@gmail.com or Ray.Norris@westernsydney.edu.au
}

Citation: Norris, R.P.; Crawford, E.; Macgregor, P. Odd Radio Circles and Their Environment. Galaxies 2021, 9 , 83. https://doi.org/10.3390/ galaxies 9040083

Academic Editors: Francesca Loi and Tiziana Venturi

Received: 11 October 2021

Accepted: 27 October 2021

Published: 28 October 2021

Publisher's Note: MDPI stays neutral with regard to jurisdictional claims in published maps and institutional affiliations.

Copyright: (C) 2021 by the authors. Licensee MDPI, Basel, Switzerland. This article is an open access article distributed under the terms and conditions of the Creative Commons Attribution (CC BY) license (https:// creativecommons.org/licenses/by/ $4.0 /)$.

\begin{abstract}
Odd Radio Circles (ORCs) are unexpected faint circles of diffuse radio emission discovered in recent wide deep radio surveys. They are typically about one arcmin in diameter, and may be spherical shells of synchrotron emission about a million light years in diameter, surrounding galaxies at a redshift of $\sim 0.2-0.6$. Here we study the properties and environment of the known ORCs. All three known single ORCs either lie in a significant overdensity or have a close companion. If the ORC is caused by an event in the host galaxy, then the fact that they tend to be in an overdensity, or have a close companion, may indicate that the environment is important in creating the ORC phenomenon, possibly because of an increased ambient density or magnetic field.
\end{abstract}

Keywords: galaxies; radio continuum

\section{Introduction}

Odd Radio Circles (ORCs) are recently discovered circles of diffuse steep-spectrum radio emission with no corresponding diffuse emission at optical, infrared, or X-ray wavelengths. The first three ORCs were found [1] in the Pilot Survey [2] of the Evolutionary Map of the Universe [3], using the Australian Square Kilometre Array Pathfinder (ASKAP) [4]. A fourth was found [2] in data from the GMRT [5] and a fifth was found, also in ASKAP data, by Koribalski et al. [6]. Figure 1 shows an ASKAP image of the first ORC to be discovered.

The ORCs superficially resemble supernova remnants (SNRs), but their Galactic latitude distribution is inconsistent with that of SNRs [1]. Similarly, other explanations involving known classes of objects (e.g., starburst rings, gravitational lenses, etc.) have also been shown to be inconsistent with the data [1]. Instead, we are forced to search for other explanations for this phenomenon.

Of the five known ORCs, three are single, and share similar parameters, and are shown in Figure 2. At the centre of each of the three single ORCs, there is a galaxy visible in optical wavelengths (see Figure 2). The probability of this happening by chance is so low as to be extremely unlikely [7]. We therefore call this galaxy the "host galaxy" of the ORC. The properties of the three single ORCs are summarised in Table 1.

The remaining two ORCs (ORCs 2 and 3), shown in Figure 3, form a pair whose centres are separated by about 2 arcmin. Since the other ORCs have a spatial sky density of $\sim 1$ per 50 sq. deg., the probability of two ORCs being located within 2 arcmin by chance is $\sim 10^{-4}$. We therefore assume they are associated, and presumably have a common origin. ORC2 has a near circular, edge-brightened, filled morphology. The diameter is $\sim 80$ arcsec. Unlike the single ORCs, the diffuse emission is not confined to the circle, but spills outside the ring. ORC3 is much fainter, appearing as a diffuse blob.

Close to ORC 2/3, there is a double-lobed AGN hosted by the galaxy WISEA J205848.80573612.1 (marked as "B" in Figure 3) at a redshift of $z \sim 0.32$. Coincident with one of the AGN jets is a bright star WISEA J205851.65-573554.1 (marked as A), which is a chance association. Radio source C is WISEA J205847.91-573653.8, an edge-on spiral galaxy at a 
redshift of $z \sim 0.28$. Any of these might be the host galaxy of the ORCs. The ORCs could perhaps be caused by an outflow from the AGN associated with B, or an outflow from the edge-on spiral galaxy C. In the centre of ORC3 is the galaxy WISEA J205856.25-573644.6 (marked D), at a redshift of $z \sim 0.62$, which might either be a chance background source, or may be an important component of this system. This system will be discussed in detail by Macgregor et al. (in preparation).

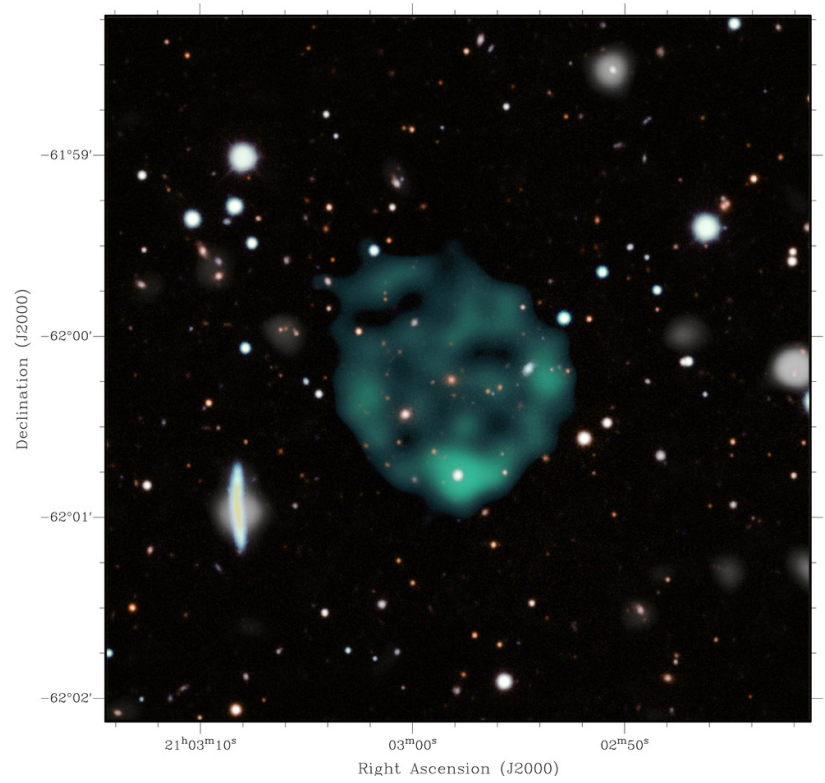

Figure 1. The ASKAP image of ORC1, adapted from Norris et al. [1]. The resolution is 11 arcsec, and the rms sensitivity is $25 \mu \mathrm{Jy} /$ beam, enhanced to show faint features, particularly the internal structure or "spokes" of the ORC. Radio data are shown in green, and the background optical image is taken from the Dark Energy Survey DR1 [8]. For the latter, the DES bands g, r, i, and z were assigned turquoise, magenta, yellow, and red, respectively, and combined using GIMP. The optical/NIR image and the radio image were then combined using a masking technique. Image credit: Jayanne English.
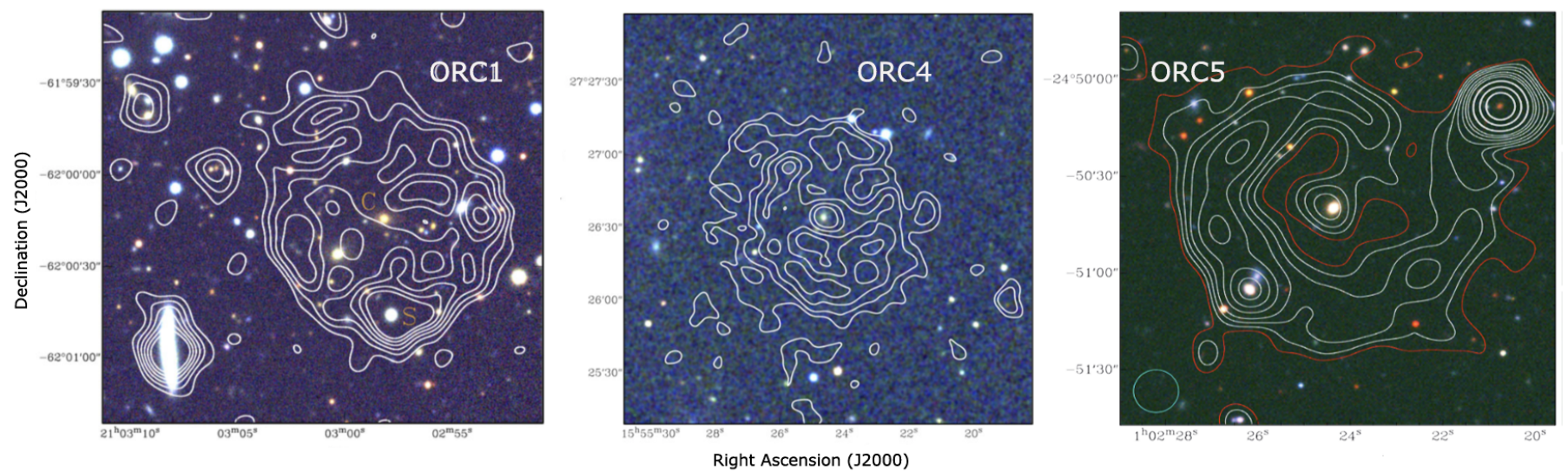

Figure 2. (Left) The ASKAP radio image of ORC1, adapted from Norris et al. [1]. The contours show the radio data (at 45, 90, 135, 180, 225, and $270 \mu \mathrm{Jy} /$ beam) overlaid onto a DES [8] 3-color composite image; DES gri-bands are coloured blue, green, and red, respectively. (Centre) The GMRT radio image of ORC 4, adapted from Norris et al. [1]. The contours show the radio data (at 150, 250, 400, 600, and $800 \mu \mathrm{Jy} /$ beam) overlaid onto a SDSS 3-colour composite image; SDSS gri-bands are coloured blue, green, and red, respectively. (Right) The ASKAP radio image of ORC5, adapted from Koribalski et al. [6]. The contours show the radio data overlaid onto a DES 3-color (grz) composite image. The dark red contour is at $65 \mu \mathrm{Jy} / \mathrm{beam}$ and remaining contours are at 90, 120, 170, 220, 270, 400, 600, and $800 \mu \mathrm{Jy} / \mathrm{beam}$. The green ellipse shows the size of the synthesised beam. 


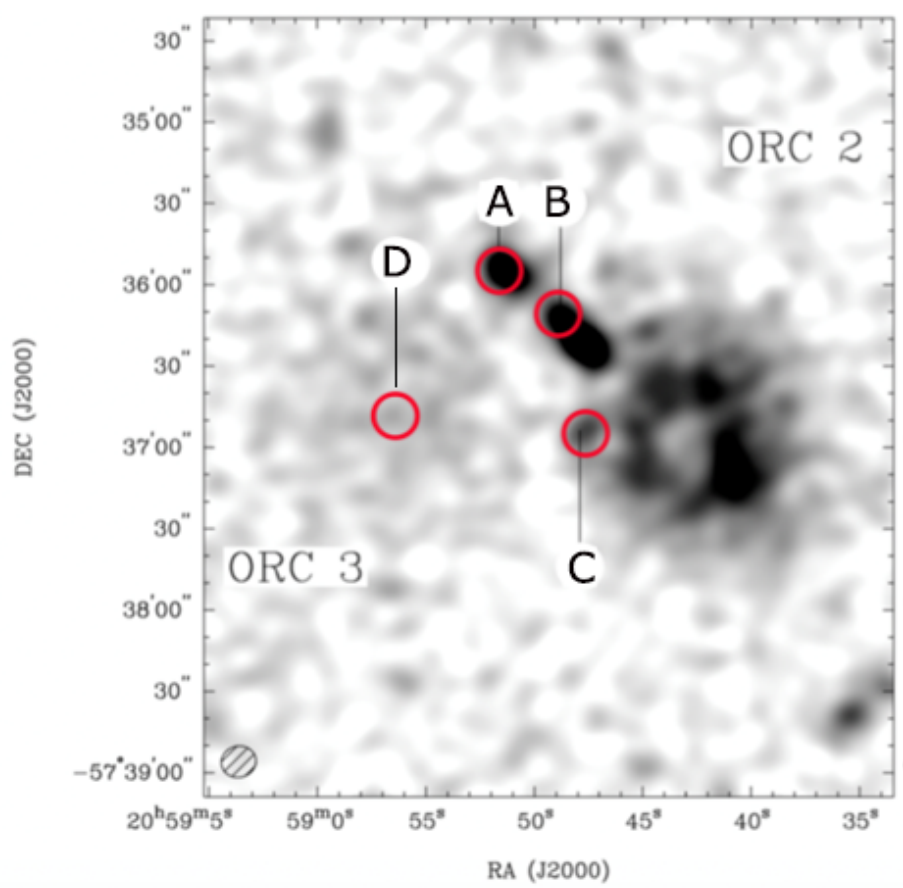

Figure 3. ASKAP radio continuum image of ORCs 2 and 3 at $944 \mathrm{MHz}$, from the EMU Pilot Survey [1], with the synthesised beam shown in the bottom left corner. ORC2 can be clearly seen on the righthand side, while ORC3 is much fainter, appearing on the left. Radio sources that correspond to putative host galaxies for ORC2 are circled in red and labelled " $\mathrm{B}$ ", “ $\mathrm{C}$ ", and " $\mathrm{D}$ ". All these galaxies have redshifts between $z \sim 0.32-0.62$. " $\mathrm{A}$ " is a bright star that is not visible in the radio image.

Table 1. Properties of the three single ORCs.

\begin{tabular}{cccccc}
\hline $\begin{array}{c}\text { Short } \\
\text { Name }\end{array}$ & IAU & RA (deg) & Dec (deg) & $\begin{array}{c}\text { Host } \\
\text { Galaxy }\end{array}$ & Redshift \\
\hline ORC 1 & ORC J2103-6200 & 315.74292 & -62.00444 & WISEA J210258.15-620014.4 & 0.551 \\
ORC 4 & ORC J1555+2726 & 238.85272 & +27.44271 & WISEA J210258.15-620014.4 & 0.457 \\
ORC 5 & ORC J0102-2450 & 15.60208 & -24.84392 & WISEA J010224.35-245039.6 & 0.270 \\
\hline
\end{tabular}

Notes: redshifts are photometric redshifts from Zou et al. [9,10]. For ORC4 we have adopted their redshift in preference to the redshift of 0.385 quoted by Norris et al. [1].

The double ORC $2 / 3$ has properties quite different from the three single ORCs, suggesting that different mechanisms are probably responsible for the two classes. Unfortunately, incomplete photometry (and hence photometric redshifts) close to ORC2/3 prevent us from including them in the analysis presented here.

In the rest of this paper we focus on the three single sources.

Norris et al. [7] argue that the rings of emission represent a spherical shell of synchrotron emission surrounding the host galaxy, and consider two hypotheses for the origin of this shell:

- it is a spherical shock wave from a cataclysmic event in the host galaxy, such as a merger of two SMBHs;

- it is the termination shock of a starburst wind from past starburst activity in the host galaxy.

Other suggested explanations for ORCs include radio jets from an AGN seen end-on (Shabala et al., in preparation) and the throats of wormholes [11,12].

In this paper we focus on the environment surrounding these host galaxies. 


\section{Environment}

To study the environment of the ORCs, we use the distribution of the photometric redshifts of the nearby galaxies, using the photometric redshift data from the Legacy Survey of the Dark Energy Spectroscopic Instrument (DESI) [9,10]. These redshifts have a typical quoted standard error of 0.05 , and comparisons (Norris, unpublished data) with spectroscopic redshifts confirm these uncertainties are reliable, with very few outliers.

In Figure 4 we show the distribution of galaxies surrounding each ORC. The top row shows the distribution of redshifts of galaxies within $1 \mathrm{arcmin}$ (or for ORC1, $0.5 \mathrm{arcmin}$ ) of the host galaxy, and the bottom row shows the projected spatial distribution of galaxies that have a redshift within \pm 0.05 of that of the host galaxy.

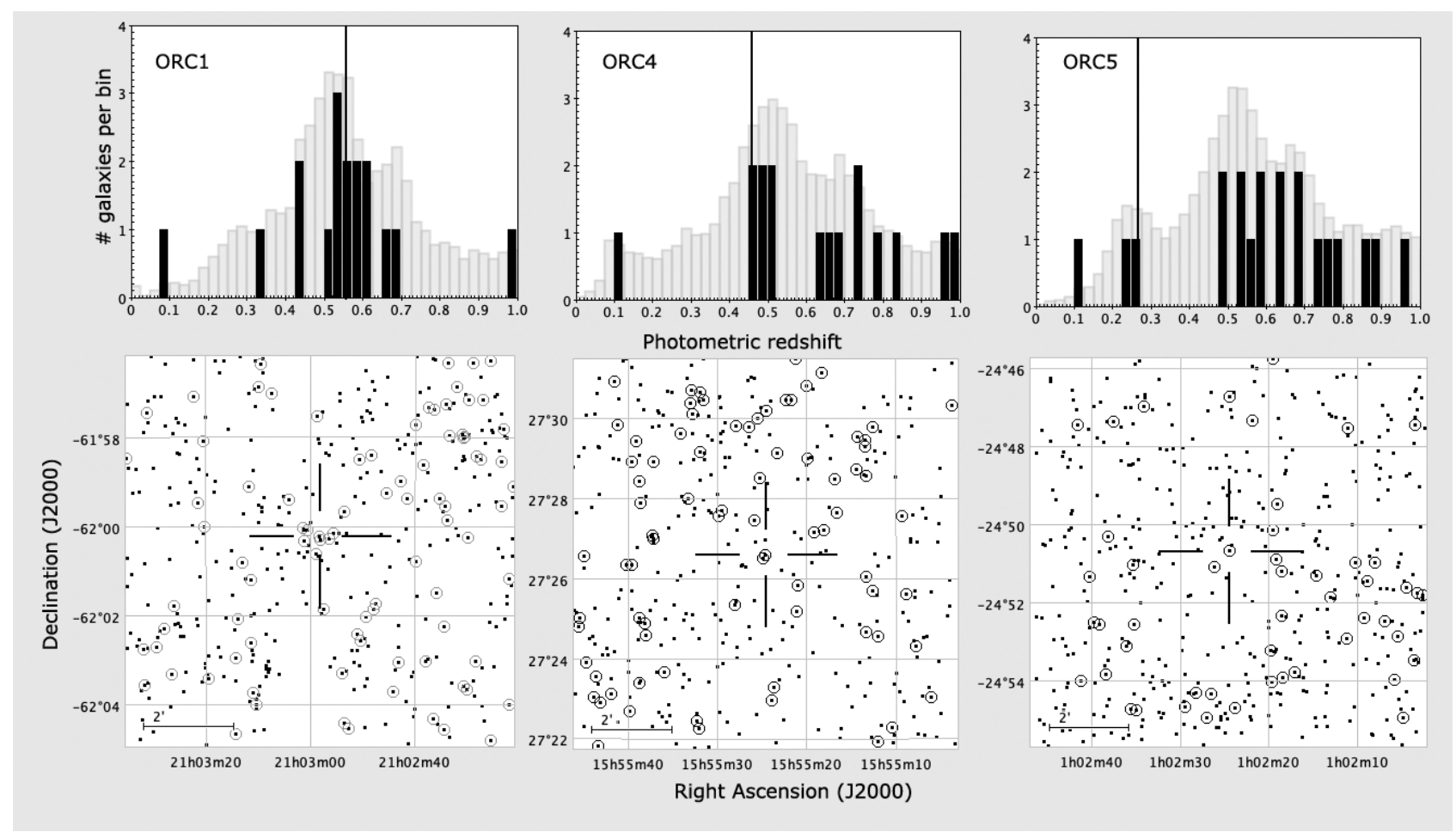

Figure 4. (Top) A histogram of the photometric redshifts [10] of the galaxies surrounding the central galaxy of each ORC. Dark columns show galaxies within 1 arcmin of the central galaxy, or in the case of ORC1, within 0.5 arcmin of the central galaxy. Grey columns show the (scaled) distribution of redshifts over a region of a few square degrees surrounding the ORC. The vertical line shows the redshift of the central galaxy. (Bottom) The galaxies surrounding each ORC. Dots show all galaxies with a photometric redshift in Zou et al. [10], and circles show those with a redshift within 0.05 of that of the central galaxy. The cross-hairs show the location of the central galaxy.

At the left of the diagram, ORC1 is clearly located in an overdensity of galaxies. There are eight galaxies with $0.5<z<0.6$ within 0.5 arcmin of the host galaxy, which gives a spatial density 9 times higher than galaxies in that redshift range in the surrounding area. We chose the radius of 0.5 arcmin to emphasise the high overdensity close to this source. The individual photometric redshifts of those eight close galaxies do not have enough precision to accurately locate them in the radial direction (i.e., along the line of sight) relative to the ORC. However, if the radial distribution is similar to the tangential (i.e., perpendicular to the line of sight) distribution, then most of these galaxies lie within a few $100 \mathrm{kpc}$ of the host galaxy, and several are presumably physically located within the shell of the ORC. The implications of this are discussed further by Norris et al. [7].

ORC4 does not exhibit a similar overdensity. However, at the centre of the lower middle plot can be seen a pair of galaxies almost coincident and at a similar redshift (i.e., the host at $15^{h} 55^{m} 24.65^{s},+27^{\circ} 26^{\prime} 33^{\prime \prime} .7, z=0.457$, and a nearby galaxy at $15^{h} 55^{m} 24.85^{s}$, 
$+27^{\circ} 26^{\prime} 29^{\prime \prime} .3, z=0.494$ ). This pair of galaxies has an apparent separation of only 6 arcsec or $35 \mathrm{kpc}$, so they are likely to be an interacting pair.

ORC5 also does not exhibit an overdensity. However, this too has a companion $\left(01^{h} 02^{m} 26.17^{s},-24^{\circ} 51^{\prime} 05^{\prime \prime} .1, \mathrm{z}=0.246\right)$ to the host galaxy $\left(01^{h} 02^{m} 24.33^{s},-24^{\circ} 50^{\prime} 39^{\prime \prime} .5\right.$, $\mathrm{z}=0.270$ ). This companion is visible in Figure 2 as the bright galaxy in the south-east limb of ORC5. This galaxy has a tangential separation of 36 arcsec or $150 \mathrm{kpc}$ from the host, but there is some indication [6] that the companion is interacting with the ORC.

\section{Discussion}

Most of the models so far proposed for ORCs [7] depend critically on the ambient density and magnetic field of the region surrounding the ORC. For example, a magnetic field comparable to the cosmic microwave background equipartition field (e.g., $8 \mu \mathrm{G}$ for ORC1 [7]) is required to generate the observed synchrotron emission. Although an ambient magnetic field will be amplified by the shock that created the ORC, an initial ambient magnetic field is still required to seed that process, and ambient magnetic fields are known to be higher in clusters and over-densities than in a non-cluster environment [7]. Thus, this model suggests that ORCs should occur preferentially in overdensity regions.

Of the three ORCs discussed here, one is located in a significant overdensity, and the other two appear to have a nearby, interacting companion. Studies (e.g., [13]) suggest that only a few percent of galaxies have an interacting companion, and so it appears that the host galaxies of ORCs are atypical. However, we are very cautious in making this claim, as (a) our sample of three is very small, and (b) the technique we have used to find companions may probe more deeply than the techniques used in large-scale searches for companions.

Nevertheless, if this apparent atypicality is supported by further work, it may offer a clue as to why ORCs are so rare (about one every 50 square degrees).

\section{Conclusions}

We have shown that each of the three known single ORCs is either located in an overdensity of nearby galaxies, or it has a close companion with which it is presumably interacting. By comparison, only a few percent of field galaxies have a known companion galaxy. We hesitate to place too much weight on a sample of three objects, but if this result is supported by further studies then it suggests an explanation as to why only one ORC is found per 50 square degrees of sky.

Author Contributions: Conceptualisation, R.P.N.; methodology, R.P.N.; data curation, R.P.N., E.C.; writing, R.P.N., E.C., P.M. All authors have read and agreed to the published version of the manuscript.

Funding: This research received no external funding.

Institutional Review Board Statement: Not applicable.

Informed Consent Statement: Not applicable.

Data Availability Statement: Not applicable.

Acknowledgments: We thank Bärbel Koribalski for comments on an early draft of this paper. This work makes use of data products from the Wide-field Infrared Survey Explorer, which is a joint project of the University of California, Los Angeles, and the Jet Propulsion Laboratory/California Institute of Technology, funded by the National Aeronautics and Space Administration. It also uses public archival data from the Dark Energy Survey (DES) and we acknowledge the institutions listed on https:/ / www.darkenergysurvey.org/the-des-project/data-access / (accessed on 26 October 2021). This research has made extensive use of the "Aladin sky atlas" (developed at CDS, Strasbourg Observatory, France [14]), TOPCAT [15] and Ned Wright's cosmology calculator [16].

Conflicts of Interest: The authors declare no conflict of interest. 


\section{References}

1. Norris, R.P.; Intema, H.T.; Kapińska, A.D.; Koribalski, B.S.; Lenc, E.; Rudnick, L.; Alsaberi, R.Z.E.; Anderson, C.; Anderson, G.E.; Crawford, E.; et al. Unexpected circular radio objects at high Galactic latitude. Publ. Astron. Soc. Aust. 2021, 38, e003. [CrossRef]

2. Norris, R.P.; Marvil, J.; Collier, J.D.; Kapinska, A.D.; O’Brien, A.N.; Rudnick, L.; Andernach, H.; Asorey, J.; Brown, M.J.I.; Bruggen, M.; et al. The Evolutionary Map of the Universe Pilot Survey. arXiv 2021, arXiv:2108.00569.

3. Norris, R.P.; Hopkins, A.M.; Afonso, J.; Brown, S.; Condon, J.J.; Dunne, L.; Feain, I.; Hollow, R.; Jarvis, M.; Johnston-Hollitt, M.; et al. EMU: Evolutionary Map of the Universe. Publ. Astron. Soc. Aust. 2011, 28, 215-248.10.1071/AS11021. [CrossRef]

4. Hotan, A.W.; Bunton, J.D.; Chippendale, A.P.; Whiting, M.; Tuthill, J.; Moss, V.A.; McConnell, D.; Amy, S.W.; Huynh, M.T.; Allison, J.R.; et al. Australian square kilometre array pathfinder: I. system description. Publ. Astron. Soc. Aust. 2021, 38, e009. [CrossRef]

5. Ananthakrishnan, S.; Pramesh Rao, A. The Indian Giant Metrewave Radio Telescope (invited). In Proceedings of the 2001 Asia-Pacific Radio Science Conference AP-RASC '01, Tokyo, Japan, 1-4 August 2001; p. 237.

6. Koribalski, B.S.; Norris, R.P.; Andernach, H.; Rudnick, L.; Shabala, S.; Filipović, M.; Lenc, E. Discovery of a new extragalactic circular radio source with ASKAP: ORC J0102-2450. Mon. Not. R. Astron. Soc. Lett. 2021, 505, L11-L15. [CrossRef]

7. Norris, R.P.; Collier, J.D.; Crocker, R.M.; Heywood, I.; Macgregor, P.; Rudnick, L.; Shabala, S.; Andernach, H.; da Cunha, E.; English, J.; et al. A detailed investigation of an Odd Radio Circle. Mon. Not. R. Astron. Soc. Lett. 2021, in preparation.

8. Abbott, T.M.C.; Abdalla, F.B.; Allam, S.; Amara, A.; Annis, J.; Asorey, J.; Avila, S.; Ballester, O.; Banerji, M.; Barkhouse, W.; et al. The Dark Energy Survey: Data Release 1. Astrophys. J. Suppl. Ser. 2018, 239, 18. [CrossRef]

9. Zou, H.; Gao, J.; Zhou, X.; Kong, X. Photometric Redshifts and Stellar Masses for Galaxies from the DESI Legacy Imaging Surveys. Astrophys. J. Suppl. Ser. 2019, 242, 8. [CrossRef]

10. Zou, H.; Gao, J.; Zhou, X.; Kong, X. VizieR Online Data Catalog: DESI photz and stellar masses galaxies (Zou+, 2019). In VizieR On-Line Data Catalog: J/ApJS/242/8. 2020. Available online: https://ui.adsabs.harvard.edu/abs/2020yCat..22420008Z/abstract (accessed on 26 October 2021)

11. Kirillov, A.A.; Savelova, E.P. Possible formation of ring galaxies by torus-shaped magnetic wormholes. Eur. Phys. J. C 2020, 80, 810. [CrossRef]

12. Kirillov, A.A.; Savelova, E.P.; Vladykina, P.O. Possible Effects of the Fractal Distribution of Relic Wormholes. Universe 2021, 7, 178. [CrossRef]

13. Yamauchi, C.; Yagi, M.; Goto, T. E+A and companion galaxies-I. A catalogue and statistics. Mon. Not. R. Astron. Soc. 2008, 390, 383-398. [CrossRef]

14. Boch, T.; Fernique, P. Aladin Lite: Embed your Sky in the Browser. In Astronomical Data Analysis Software and Systems XXIII; Astronomical Society of the Pacific Conference Series; Manset, N., Forshay, P., Eds.; Astronomical Society of the Pacific: San Francisco, CA, USA, 2014; Volune 485, p. 277.

15. Taylor, M.B. TOPCAT \& STIL: Starlink Table/VOTable Processing Software. In Astronomical Data Analysis Software and Systems XIV; Astronomical Society of the Pacific Conference Series; Shopbell, P., Britton, M., Ebert, R., Eds.; Astronomical Society of the Pacific: San Francisco, CA, USA, 2005; Volume 347, p. 29.

16. Wright, E.L. A Cosmology Calculator for the World Wide Web. Publ. Astron. Soc. Pac. 2006, 118, 1711-1715. [CrossRef] 